\title{
Event Rates of Gravitational Waves from merging Intermediate- mass Black Holes: based on a Runaway Path to a SMBH
}

\author{
Hisaaki Shinkai ${ }^{1, \star}$ \\ ${ }^{1}$ Department of Information Systems, Osaka Institute of Technology, Hirakata City, Osaka 573-0196, Japan
}

\begin{abstract}
Based on a dynamical formation model of a supermassive black hole (SMBH), we estimate the expected observational profile of gravitational wave at ground-based detectors, such as KAGRA or advanced LIGO/VIRGO. Noting that the second generation of detectors have enough sensitivity from $10 \mathrm{~Hz}$ and up, we are able to detect the ring-down gravitational wave of a BH with the mass $M<2 \times 10^{3} M_{\odot}$. This enables us to check the sequence of $\mathrm{BH}$ mergers to $\mathrm{SMBHs}$ via intermediate-mass $\mathrm{BHs}$. We estimate the number density of galaxies from the halo formation model and estimate the number of $\mathrm{BH}$ mergers from the giant molecular cloud model assuming hierarchical growth of merged cores. At the designed KAGRA (and/or advanced LIGO/VIRGO), we find that the BH merger of its total mass $M \sim 60 M_{\odot}$ is at the peak of the expected mass distribution. With its signal-to-noise ratio $\rho=10(30)$, we estimate the event rate $R \sim 200(20)$ per year in the most optimistic case, and we also find that BH mergers in the range $M<150 M_{\odot}$ are $R>1$ per year for $\rho=10$. Thus, if we observe a $\mathrm{BH}$ with more than $100 M_{\odot}$ in future gravitational-wave observations, our model naturally explains its source.
\end{abstract}

\section{Introduction}

The LIGO group reported three detections of gravitational waves (GW150914, GW151226, GW170104) and one transient event (LVT151012) so far (see Table. 1), all three of which are regarded as the events of coalescence of binary black holes (BBHs). These announcements were not only valuable on the point of the direct detections of the gravitational wave, but also the first results of confirming the existence of $\mathrm{BHs}$, the existence of BHs of this mass range, and the existence of BBHs.

In this article, as a possible sources of $\mathrm{BHs}$ of $30 M_{\odot}-60 M_{\odot}$, we consider simple sequences of mergers of $\mathrm{BHs}$, which start from $10 M_{\odot} \mathrm{BHs}$ and end with a supermassive $\mathrm{BH}(\mathrm{SMBH})$ via intermediate-mass BHs (IMBHs). After counting the numbers of galaxies in the Universe from a halo formation model, and modeling the numbers of BHs in a galaxy from a giant molecular cloud scenario, we estimate their observational detectability at ground-based gravitational-wave detectors. More details are reported in [1].

The formation process of an SMBH is one of the unsolved problems in galaxy evolution history. Many possible routes were suggested by Rees [5] long ago, but we still debate a plausible route. In this article, we take the scenario that SMBHs are formed by accumulations of BHs. This route was came to be believed when an IMBH $\left(10^{2}-10^{3} M_{\odot}\right)$ was first discovered in a starburst galaxy M82. So far, many IMBHs have been found in the center of galaxies.

\footnotetext{
${ }^{\star}$ e-mail: hisaaki.shinkai@oit.ac.jp
} 
Table 1. GW events (as of July 2017)

\begin{tabular}{|c|c|c|c|c|c|c|c|c|}
\hline & \multicolumn{2}{|c|}{ Primary \& Secondary } & \multicolumn{3}{|c|}{ Final BH } & SNR & $\mathrm{Mpc}$ & $\operatorname{deg}^{2}$ \\
\hline & $m_{1} / M_{\odot}$ & $m_{2} / M_{\odot}$ & $M_{\text {final }} / M_{\odot}$ & $\Delta M / M_{\odot}$ & $a / M_{\odot}$ & $\rho$ & $z$ & \\
\hline GW150914 & 36.2 & 29.1 & 62.3 & -3 & 0.68 & 24 & $410 \mathrm{Mpc}$ & 600 \\
\hline ref [2] & $\begin{array}{l}+5.2 \\
{ }_{-3.8}\end{array}$ & $\begin{array}{l}+3.7 \\
-4.4\end{array}$ & $\begin{array}{l}+3.7 \\
{ }_{-3.1}\end{array}$ & $(4.59 \%)$ & & & 0.09 & \\
\hline LVT151012 & 23 & 13 & 35 & -1 & 0.66 & & & \\
\hline & $\begin{array}{l}+18 \\
-6\end{array}$ & $\begin{array}{l}+4 \\
-5\end{array}$ & $\begin{array}{l}+14 \\
-4\end{array}$ & $(2.78 \%)$ & & & & \\
\hline GW151226 & 14.2 & 7.5 & 20.8 & -0.9 & 0.74 & 13 & $440 \mathrm{Mpc}$ & \\
\hline ref [3] & +8.3 & +2.3 & ${ }_{-17}^{+6.1}$ & $(4.15 \%)$ & & & 0.09 & \\
\hline GW170104 & 31.2 & 19.4 & 48.7 & -1.9 & 0.64 & 13 & $880 \mathrm{Mpc}$ & 1300 \\
\hline $\operatorname{ref}[4]$ & $\begin{array}{l}+8.4 \\
{ }_{-60}\end{array}$ & $\begin{array}{r}+5.3 \\
-59\end{array}$ & +5.7 & $(3.75 \%)$ & & & 0.18 & \\
\hline
\end{tabular}

This runaway path was first proposed by Ebisuzaki et al.[6]. The scenario consists of three steps: (1) formation of IMBHs by runaway mergers of massive stars in dense star clusters, (2) accumulations of IMBHs at the center region of a galaxy due to sinkages of clusters by dynamical friction, and (3) mergers of IMBHs by multibody interactions and gravitational radiation. Successive mergers of IMBHs are likely to form an SMBH with a mass of heavier than $>10^{6} M_{\odot}$. Numerical simulations support the above first step and the second step is also confirmed in a realistic mass-loss model, while the third step is not yet investigated in detail. The discovery of an SMBH binary system, together with a simulation of an eccentric evolution of SMBH binaries, supports this formation scenario through merging of IMBHs. (See references in [1]).

In Matsubayashi et al.(2004)[7], we pointed out that gravitational waves from IMBHs can be a trigger to prove this process. Later, Fregeau et al.[8] discussed the event rates of IMBH-IMBH binary observations at advanced LIGO and VIRGO and concluded that we can expect $\sim 10$ mergers per year. This work was followed by Gair et al.[9], including the Einstein Telescope project. Amaro-Seoane \& Santamaría [10] also discussed the IMBH-IMBH system, including the pre-merger phase.

\section{Black Hole Merger Model}

\section{Ring-down Frequency from $\mathrm{BHs}$}

The gravitational waveform of binary-star mergers which ends up with a single $\mathrm{BH}$, has three typical phases: inspiral phase, merging phase, and ring-down phase. The ring-down frequency is simply estimated by the quasi-normal frequency of $\mathrm{BHs}$, which is determined from the mass and spin of the final $\mathrm{BH}$ and is estimated to be higher frequency than in its inspiral phase.

Supposing that advanced GW interferometers can detect $f_{\mathrm{qnm}}$ above $10 \mathrm{~Hz}$, then BHs less than $1200 M_{\odot}$ are within the target if BHs are nonrotating ( $\left.a=0\right)$, while BHs less than $2500 M_{\odot}$ are in the detectable range for highly rotating cases $(a=0.98)$.

\section{Number of Galaxies in the Universe}

In order to model the typical mass of galaxies and its distribution, we apply the halo mass function given by Vale \& Ostriker [11], in which they discuss an empirically based, nonparametric model for galaxy luminosities with halo/subhalo masses. They apply the Sheth-Tormen mass function for halo number density. Integrating it by the volume as a function of redshift $z$ (we use the standard cosmology model), we can derive the number density of halos (Figure 1(left)). Combining the average number of galaxies and the number density of halos, we get the number density of galaxies $n_{\text {galaxy }}(M, z)$, which we show in Fig.1(right). 

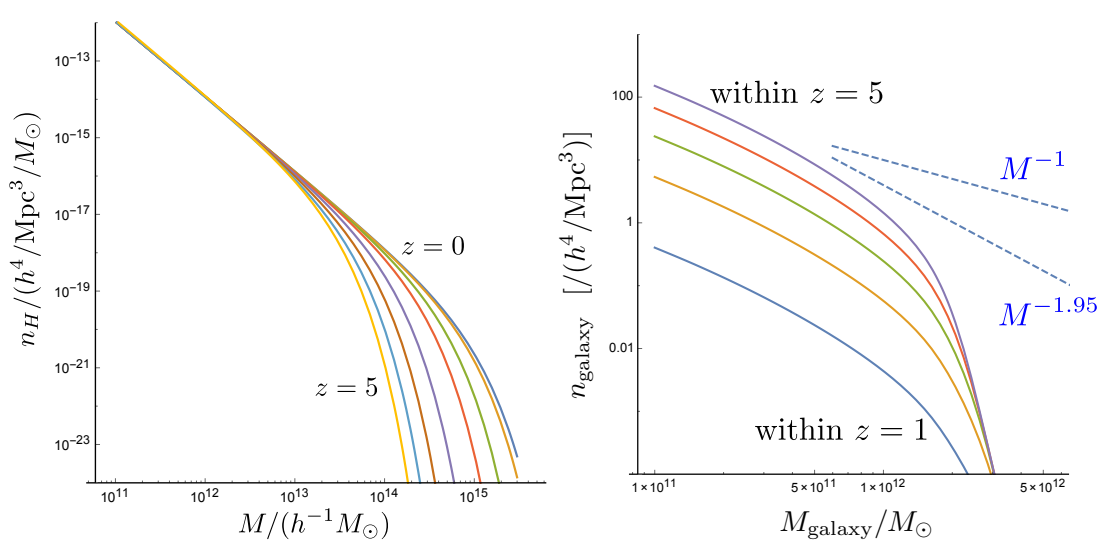

Figure 1. (left) Global mass functions for halos (halo and subhalo), $n_{H}(M)$, for $z=0,0.1,0.5,2,4,5 . n_{H}(M)$ is in units of $h^{4} / \mathrm{Mpc}^{3} / M_{\odot} . M$ is in units of $h^{-1} M_{\odot}$, with $h=0.7$. (right) Number density of galaxies, $n_{\text {galaxy }}(M)$.

\section{Number of BHs in a Galaxy}

We next estimate the number of BH candidates in a galaxy. Recently, Inutsuka et al. [12] developed a scenario of galactic-scale star formation from a giant molecular cloud. Their model includes both the growth of molecular clouds and the destruction of magnetized molecular clouds by radiation. Simulations and steady-state analysis show that the mass density function of molecular clouds, $n_{\mathrm{cl}}\left(M_{g}\right)$, converges at the Schechter-like function, $n_{\mathrm{cl}}\left(M_{\mathrm{cl}}\right) \sim M_{\mathrm{cl}}^{-1.7} \exp \left(-M_{\mathrm{cl}} / M_{\text {cut }}\right)$ where the cutoff mass $M_{\text {cut }}=10^{6} M_{\odot}$. On the other hand, many $N$-body simulations report that there is a simple relation between the mass of the most massive cluster $m_{\max }$ and the total mass of the molecular cloud $M_{\mathrm{cl}}$, $m_{\max }=0.20 M_{\mathrm{cl}}^{0.76}$.

We therefore combine these results, and we suppose that each molecular cloud forms a single $\mathrm{BH}$ in its core if it is more than $10 M_{\odot}$, and we suppose that these BHs become "building blocks" for forming stellar-sized and intermediate-mass BHs. Many $N$-body simulations suggest that massive objects will accumulate in the center of a galaxy owing to dynamical friction, so that we modeled that these seed BHs accumulate and merge repeatedly (as we model below), resulting in IMBHs and SMBHs. We do not specify where these mergers occur, but we count our BH mergers after we set up the initial seeds. We show the number density of BHs in a galaxy, $n_{\mathrm{BH}}\left(M_{\mathrm{BH}}\right)$ in Fig. 2(left).
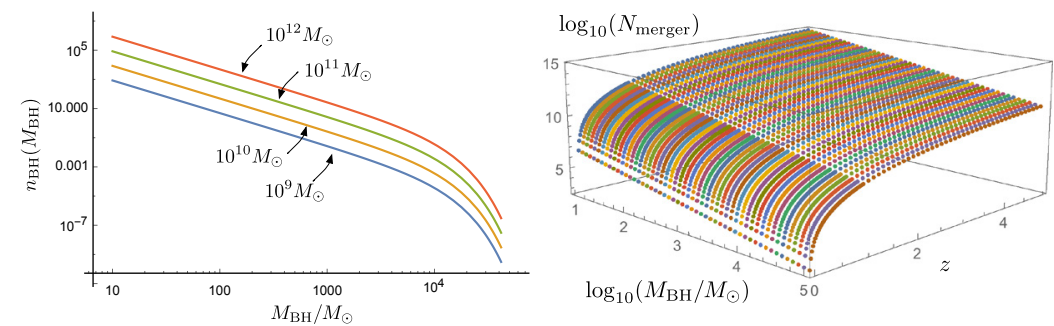

Figure 2. (left) Number density of BHs per galaxy as a function of $\mathrm{BH}$ mass for different total mass of galaxies $M_{\text {galaxy }}=10^{9} M_{\odot}-10^{12} M_{\odot}$. (right) Cumulative distribution function of the number of $\mathrm{BH}$ mergers $N_{\text {merger }}\left(M_{\mathrm{BH}}\right)$ as a function of the redshift $z$. We express number with binned one, of which we binned 20 for one order in $M_{\mathrm{BH}}$.

\section{Number of BH Mergers in a Galaxy}

We simply assume that BHs formed at cores of clouds will accumulate each other hierarchically, i.e. the mass and the number of BHs at steps from $k$ to $k+1$ can be expressed simply by $M_{k+1}=2 M_{k}$, and $N_{k+1}=N_{k} / 2$. The mass of a BH merger, then, obeys the distribution $M^{-1}$. On the other hand, we know empirically that the mass of the central $\mathrm{BH}$ of the galaxy, $M_{\mathrm{SMBH}}$, and the total mass of the galaxy, $M_{\text {galaxy }}$, has a relation $M_{\mathrm{SMBH}}=2 \times 10^{-4} M_{\text {galaxy }}$ (or equal to $10^{-3}$ of the bulge mass). 
Combining these facts, for a certain galaxy with $M_{\text {galaxy }}$, we pick up BHs with total mass $M_{\mathrm{SMBH}}$ (equation above), obeying the mass distribution of Fig.2 (left). We suppose that picked-up BHs will form an SMBH in its series of mergers in the hierarchical model. Together with galaxy distribution function $n_{\text {galaxy }}(M, z)$, we are able to count the possible events of $\mathrm{BH}$ mergers, $N_{\text {merger }}\left(M_{\mathrm{BH}}, z\right)$, in the universe, which we show in Fig.2 (right).

\section{Signal-to-noise Ratio, Detectable Distance, and Event Rate}

The standard procedure for the detection is judged by the optimal $\mathrm{S} / \mathrm{N}$ ratio, $\rho$, which is given by

$$
\rho=2\left[\int_{0}^{\infty} \frac{\tilde{h}(f) \tilde{h}^{*}(f)}{S_{n}(f)} d f\right]^{1 / 2},
$$

where $\tilde{h}(f)$ is the Fourier-transformed quantity of the wave, and $S_{n}(f)$ is the (one-sided) power spectral density of strain noise of the detector. For KAGRA (bKAGRA), we use a fitted function

$$
\sqrt{S_{n}(f)}=10^{-26}\left(6.5 \times 10^{10} f^{-8}+6 \times 10^{6} f^{-2.3}+1.5 f\right),
$$

where $f$ is measured in $\mathrm{Hz}$, as was used in [13].

Following Flanagan $\&$ Hughes [14], we use the energy spectrum formula for the ring-down wave which results in $E_{\text {ringdown }} \approx \frac{1}{8} A^{2} M^{2} f_{\text {ringdown }} Q$, where $M$ is the total mass of the binary, $M=m_{1}+m_{2}$.

Let $\epsilon_{r}(a) \equiv E_{\text {ringdown }} / M$, which expresses the energy fraction of the emitted gravitational wave to the total mass. As we cited in the introduction, three GW events suggest us that the $4.0 \%$ of the total mass is emitted before the merger. The associate numerical simulations show that the ring-down part emits the energy around $0.6 \%$ of the total mass. If we use $A \sim 0.4$, then we recover the ratio $\epsilon_{r}(0.67)=0.58 \%$. The magnitude of this $A$ is also consistent with the quadrupole formula.

The $\mathrm{S} / \mathrm{N}$ is, then, expressed using the inertial mass $\mu=m_{1} m_{2} / M$ and the redshift of the source $z$,

$$
\rho^{2}=\frac{8}{5} \frac{\epsilon_{r}(a)}{f_{R}^{2}} \frac{(1+z) M}{S_{h}\left(f_{R} /(1+z)\right)}\left(\frac{(1+z) M}{d_{L}(z)}\right)^{2}\left(\frac{4 \mu}{M}\right)^{2} .
$$

By specifying the $\mathrm{BH}$ mass and spin, together with $\rho$, we can then find the distance $d_{L}$ that satisfies eq. (3). We call this distance the detectable distance, $D(M, a, \rho)$. We show them in Fig.3(left) for KAGRA for $\mathrm{S} / \mathrm{R}=10$ and 100 . Using $D(M, a, \rho)$, we set the upper limit of $z$ for integrating the number of galaxies, $N_{\text {galaxy }}$, and then obtain the number of BH mergers, $N_{\text {merger }}$. We show $N_{\text {merger }}$ in Figures 3 (a1) and (b1) for $\mathrm{S} / \mathrm{R}=10$ and 30 , respectively.

The event rate $R$, then, is estimated by $R[/ \mathrm{yr}]=N_{\text {merger }}(z) / V(D / 2.26)$, where the factor 2.26 is for averaging the distance for all directions. We show them in Figs 3 (a2) and (b2). These figures are for specifying the $\mathrm{BH}$ spin parameter $a$, but if we assume that $a$ is homogeneously distributed, then the averaged $R$ is estimated as its peak $R \sim 7.13[/ \mathrm{yr}]$ at $M \sim 59.1 M_{\odot}(200-375[\mathrm{~Hz}]$ for $a=0-0.9)$. The mergers of the range above $R>1$ [/yr] have mass $40 M_{\odot}<M<150 M_{\odot}$. The total number of events above $R>1[/ \mathrm{yr}]$ is $\sim 211$.

\section{Summary}

Our event rate sounds similar to that of other groups. For example, the LIGO-Virgo group updated their estimated event rates after the detection of GW150914 as 2-600 $\mathrm{Gpc}^{3} \mathrm{yr}^{-1}$ assuming BH mass distribution models as flat or power law ( $M^{-2.35}$; [15]). Kinugawa et al. [16] estimate as 70-140 


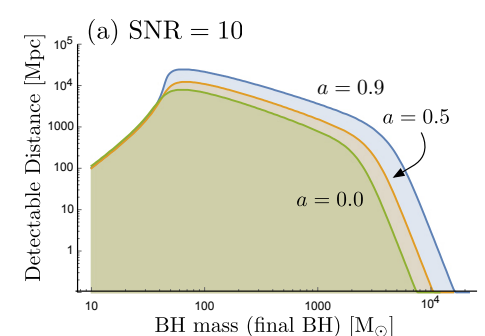

(b) $\mathrm{SNR}=100$

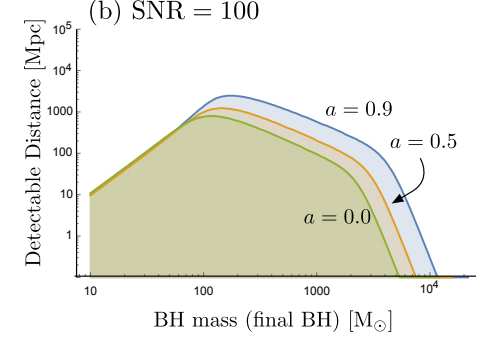

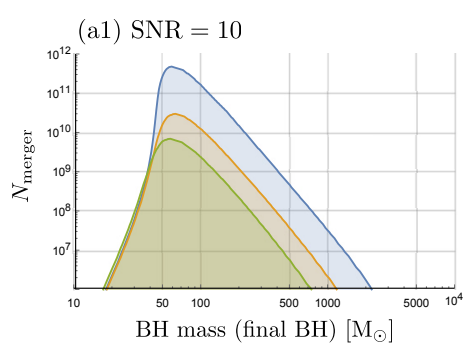

(b1) $\mathrm{SNR}=30$

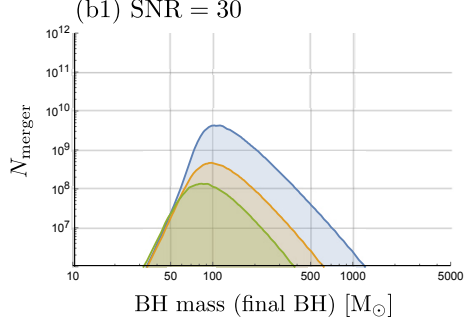

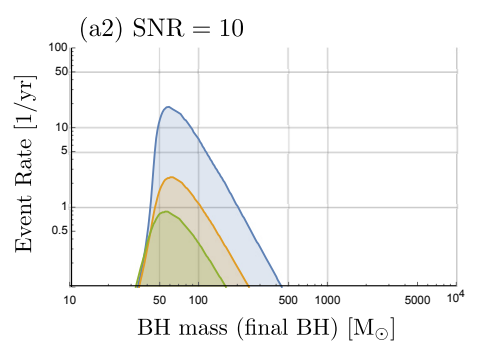

(b2) $\mathrm{SNR}=30$

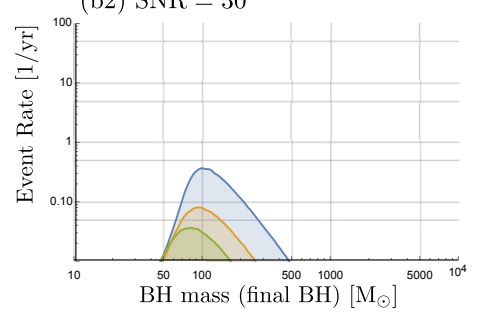

Figure 3. (left) Detectable distance $D$ of the ring-down signal at KAGRA. S/R is set to (a) 10 and (b) 100. (middle and right) Number of BH mergers within the detectable distances (a1, b1) and event rate $R$ (a2, b2) as a function of $\mathrm{BH}$ mass $M$ with $\mathrm{S} / \mathrm{N}$ ratio $\rho=10$ and 30 for KAGRA. Three distributions for each figure are of $a=0.9,0.5$, and 0.0 (from largest to lowest), respectively.

$\mathrm{yr}^{-1}$ from their Population III model. Inoue et al.[17] estimate as $<60 \mathrm{yr}^{-1}$ from their $\mathrm{BH}$ merger model inferred from the luminosity function of ultraluminous X-ray sources. However, our model predicts $\mathrm{BH}$ mergers with $M>100 M_{\odot}$, which will be a key to test our model in the future.

This work was supported in part by the Grant-in-Aid for Scientific Research Fund of the JSPS (C) No. 25400277, MEXT KAKENHI Grants No. 17H06357 and No. 17H06358.

\section{References}

[1] Shinkai, H., Kanda, N., and Ebisuzaki, T., Astrophys. J. 835 (2017) 276.

[2] Abbott, B. et al. Phys. Rev. Lett. 116 (2016) 061102.

[3] Abbott, B. et al. Phys. Rev. Lett. 116 (2016) 241103.

[4] Abbott, B. P. et al. Phys. Rev. Lett. 118 (2017) 221101.

[5] Rees, M. J. Observatory 98(1978) 210; Annu. Rev. Astron. Astrophys. 22(1984) 471.

[6] Ebisuzaki, T., et al., 2001, Astrophys. J. Lett. 562, L19.

[7] Matsubayashi, T., Shinkai, H., \& Ebisuzaki, T., Astrophys. J. 614 (2004) 864.

[8] Fregeau J. M., et al. Astrophys. J. 646 (2006) L135.

[9] Gair, J. R., Gen. Rel. Grav. 43 (2011) 485.

[10] Amaro-Seoane, P., \& Santamaría, L., Astrophys. J. 722 (2010) 1197.

[11] Vale, A., \& Ostriker, J.P., Mon. Not. Roy. Astro. Soc. 371 (2006) 1173.

[12] Inutsuka, S., Inoue, T., Iwasaki, K. \& Hosokawa, T., Astro. Astrophys. 580 (2015) A49.

[13] Nakano, H., Tanaka, T., \& Nakamura, T., Phys. Rev. D 92 (2015) 064003.

[14] Flanagan, É. É., \& Hughes. S. A., Phys. Rev. D 57 (1998) 4535.

[15] Abbott, B. et al. Astrophys. J. Lett. 833 (2016) 1.

[16] Kinugawa, T., et al. Mon. Not. Roy. Astro. Soc. 442 (2014) 2963.

[17] Inoue, Y., Tanaka, Y.T., \& Isobe, N., Mon. Not. Roy. Astro. Soc. 461 (2016) 4329. 